\title{
Clinicopathological features of the intraductal papillary neoplasms of the intrahepatic bile duct
}

\author{
Gi-Woong Jang, Shin Hwang, Yong-Joo Lee, Ki-Hun Kim, Kwang-Min Park, Chul-Soo Ahn, \\ Deok-Bog Moon, Tae-Yong Ha, Gi-Won Song, Dong-Hwan Jung, Gil-Chun Park, and Sung-Gyu Lee
}

Department of Surgery, Asan Medical Center, University of Ulsan College of Medicine, Seoul, Korea

\begin{abstract}
Backgrounds/Aims: This study is intended to investigate the clinicopathological features of the intraductal papillary neoplasms of the intrahepatic bile duct (IPNB), especially focused on malignant changes. Methods: From the institutional database of liver resection cases (Asan Medical Center, University of Ulsan College of Medicine), 18 patients who met the definition of IPNB were selected. They had undergone liver resection between February 2002 and October 2006; thus, the follow-up period was more than 5 years. Results: Of the 18 patients, 11 patients were male. Their mean age was $61.3 \pm 6.7$ years. There were no differences between the non-malignant and malignant lesions, in the comparison of the CEA levels $(5.6 \pm 2.7$ vs. $12.6 \pm 31.1 \mathrm{ng} / \mathrm{ml}, p=0.439)$ and the CA19-9 levels $(29.2 \pm 34.7$ vs.31.9 \pm 30.2 $\mathrm{ng} / \mathrm{ml}, p=0.871$ ). The common radiologic findings were: intraductal growing mass in 10; bile duct dilatation in 6; and saccular duct dilatation in 2. Left and right hepatectomies were performed in 15 and 3, respectively. Five patients showed benign lesions of IPNB, and 13 patients revealed malignant lesions of intraductal papillary adnocarcinoma or cholangiocarcinoma. All 4 patients with benign lesions survived for a mean period of 53 months without recurrence. In 13 patients with the malignant lesions, 1-year, 3-year, and 5-year survival rates were $100 \%, 84.6 \%$, and $59.2 \%$, respectively. Conclusions: We concluded that intrahepatic IPNB is a rare type of biliary neoplasm which includes a histological spectrum, ranging from benign disease to invasive malignancy. The long-term survival was anticipated after complete curative resection. (Korean J Hepatobiliary Pancreat Surg 2012;16:138-141)
\end{abstract}

Key Words: Intraductal papillary neoplasm; Intrahepatic duct; Resection

\section{INTRODUCTION}

The intraductal papillary neoplasms of the bile duct (IPNB) has recently referred to certain types of papillary tumours occurring in the extrahepatic and intrahepatic bile ducts with malignant potentials. ${ }^{1-3}$ The papillary tumours are also termed biliary papillomatosis, papillary adenomas, and papillary cholangiocarcinoma. ${ }^{4}$ IPNB has rarely been reported in the literature. Both benign and malignant forms of biliary papillary neoplasm are classified by the WHO. ${ }^{5}$ IPNB has also been proposed as the biliary counterpart of the intraductal papillary mucinous neoplasms of the pancreas. ${ }^{6,7}$ This study intended to evaluate the clinicopathological features of IPNB occurring in the intrahepatic ducts.

\section{METHODS}

\section{Definition of the intraductal papillary \\ neoplasms of the bile duct (IPNB)}

According to Naito et al. ${ }^{8}$ IPNB was defined as: a tumor that was localized in the liver; arose within the intrahepatic bile duct; had a major lesion that was non-infiltrative and showed an intraductal proliferation pattern; and clinicopathologically communicated with the surrounding bile duct.

\section{Patient selection}

From our institutional database of liver resection cases, 18 patients who met the definition of intrahepatic IPNB were selected. They underwent liver resection between February 2002 and October 2006, thus the follow-up peri-

Received: October 3, 2012; Revised: October 20, 2012; Accepted: October 22, 2012

Corresponding author: Shin Hwang

Department of Surgery, Asan Medical Center, University of Ulsan College of Medicine, 388-1, Poongnap-dong, Songpa-gu, Seoul 138-736, Korea

Tel: +82-2-3010-3930, Fax: +82-2-3010-6701, E-mail: shwang@amc.seoul.kr 
od was more than 5 years. Their medical records were reviewed retrospectively following the approval by the Institutional Review Board of the institution.

\section{Preoperative cholangioscopic evaluation}

For the percutaneous transhepatic cholangioscopy (PTCS), the initial percutaneous transhepatic biliary drainage (PTBD) was performed by using a pigtail catheter under fluoroscopic guidance. Two to 3 days after PTBD, the sinus tract was dilated. PTCS was performed 7 days after the tract dilatation. This interval was required for the stabilization and maturation of the sinus tract. Cholangioscopic evaluation was performed with a cholangioscope, with either a 4.9-mm external dimension (FCN-15X; Pentax, Tokyo, Japan) or a 5.1-mm external dimension (FCN1530; Pentax). During the cholangioscopic evaluation, the various mucosal appearances of the bile duct tumors and strictures were studied. Multiple targeted biopsies were performed with forceps (KA 1811S, Pentax) under direct cholangioscopic visualization. $^{9}$

\section{Statistics}

Numerical variables were presented as means with standard deviations or as medians with ranges. Survival and recurrence rates were determined by the KaplanMeier method and compared by the log-rank test. A value of $p<0.05$ was considered to be statistically significant.

\section{RESULTS}

\section{Demographic data and clinical characteristics}

Of 18 patients, 11 patients were male. Their mean age was $61.3 \pm 6.7$ years (range: 47-78). The initial clinical manifestations included: abdominal pain or discomfort in 6; gastrointestinal symptoms in 5; and incidental detection in 7. Prior cholecystectomy had been performed in 6 patients due to gallstone disease. Jaundice was accompanied in 3 patients. Intrahepatic duct stone was combined in 6 patients. No patient was associated with clonorchiasis infestation. Mean carcinoembryonic antigen (CEA) level was $8.9 \pm 25.5 \mathrm{ng} / \mathrm{ml}$ (range: 0.5-101). Mean CA19-9 level was $31.1 \pm 30.5 \mathrm{ng} / \mathrm{ml}$ (range: $2.7-90.5$ ). There were no differences between the non-malignant and malignant lesions in the comparison of the CEA levels $[5.6 \pm 2.7$ vs. $12.6 \pm$ $31.1, p=0.439$ (likely due to the small sample number)] and CA19-9 levels (29.2 \pm 34.7 vs. $31.9 \pm 30.2, p=0.871)$.

\section{Radiological findings}

All patients underwent radiological examinations, including abdomen ultrasonography (US), computed tomography $(\mathrm{CT})$, and magnetic resonance imaging (MRI) with magnetic resonance cholangiopancreatography (MRCP). Endoscopic retrograde cholangio-pancreatography (ERCP) was performed in 3 patients. Preoperative PTCS evaluation was performed in 8 patients, and the malignant cells were diagnosed in 4 (50\%). The common radiologic findings were: intraductal growing mass in 10, bile duct dilatation in 6 , and saccular duct dilatation in 2 .

\section{Surgical procedures}

Tumor locations were the left liver in 15 and the right liver in 3. Thus the operations were: left hepatectomy in 7 , left hepatectomy plus caudate resection in 1, left hepatectomy plus caudate resection with bile duct resection in 4 , left lateral sectionectomy in 1 , right hepatectomy in 2 , right hepatectomy plus caudate resection with bile duct resection in 1 , and right posterior sectionectomy in 1 . One patient underwent right hepatectomy after preoperative portal vein embolization.

Early surgical complications were abdominal abscess requiring percutaneous drainage in 2 and wound infection in 2 .

\section{Pathological findings}

Five patients showed benign lesions of IPNB, but 13 patients revealed malignant lesions of intraductal papillary adnocarcinoma or cholangiocarcinoma (Fig. 1). In 13 patients with malignant lesions, 8 showed confined tumour extents within the bile duct, and the other 5 showed periductal or hepatic parenchymal invasion. Mean maximal diameter of the tumour was $4.1 \pm 2.2 \mathrm{~cm}$ (range: $2.0-11.2$ ). The bile duct resection margin was tumour cell-positive in 2 patients. No patient showed lymph node metastasis.

\section{Survival outcomes}

All 4 patients with benign lesions survived for a mean period of 53 months (range: 25-85) without recurrence.

Among the 13 patients with malignant lesions, adjuvant chemotherapy and radiotherapy were performed in 4 patients. Concurrent adjuvant chemotherapy and radio- 

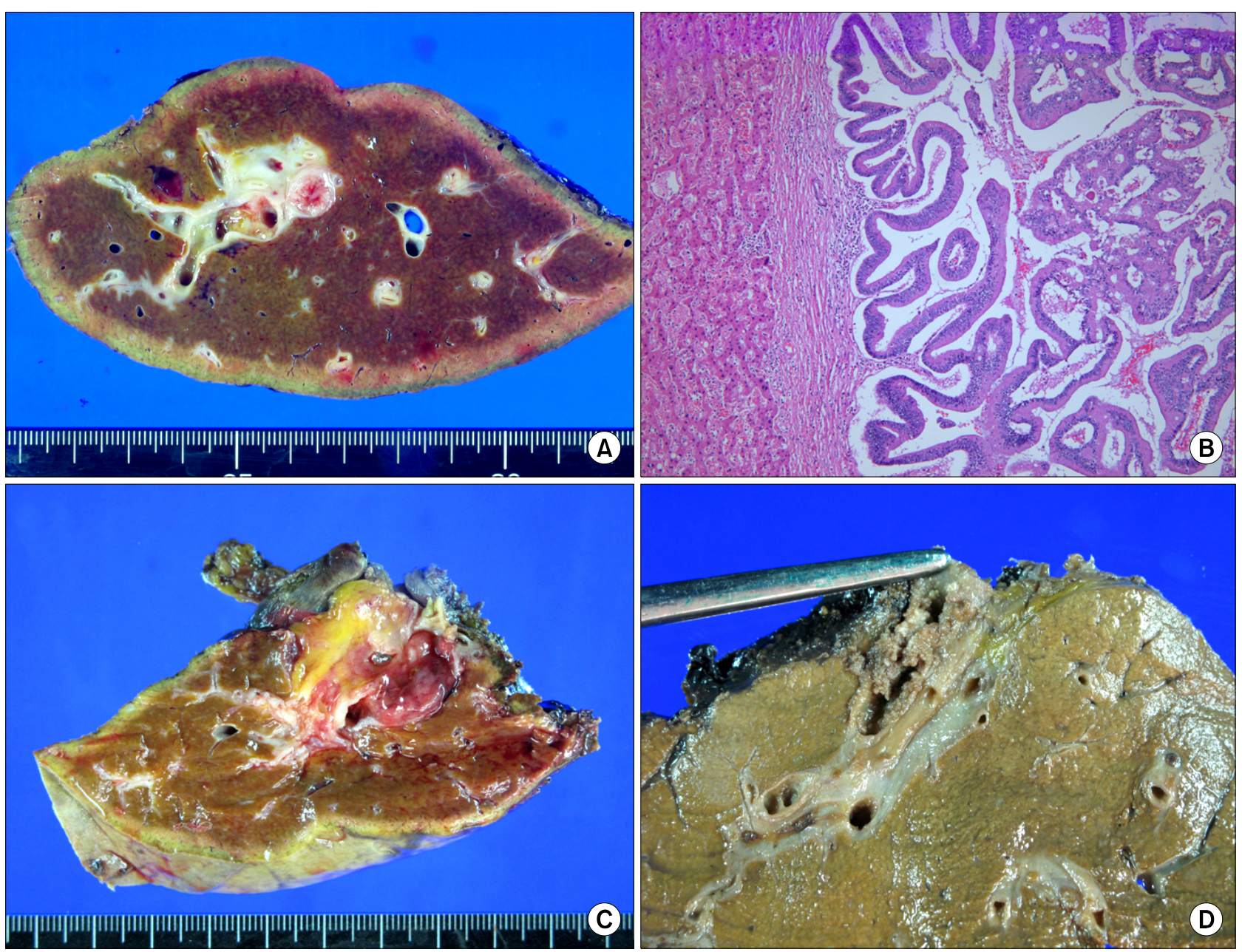

Fig. 1. Liver specimen photographs. (A) Resected left liver shows intraductal papillary neoplasm. (B) This low-grade dysplasia lesion shows no liver parenchymal invasion and no lymphovascular emboli (H\&E, $\times 100)$. (C) Resected left liver shows well-differentiated intraductal papillary adenocarcinoma with minimal periductal invasion and no lymphovascular invasion. (D) Resected right liver shows intraductal papillary adenocarcinoma with extension to the subepithelial connective tissue and lymphovascular invasion.

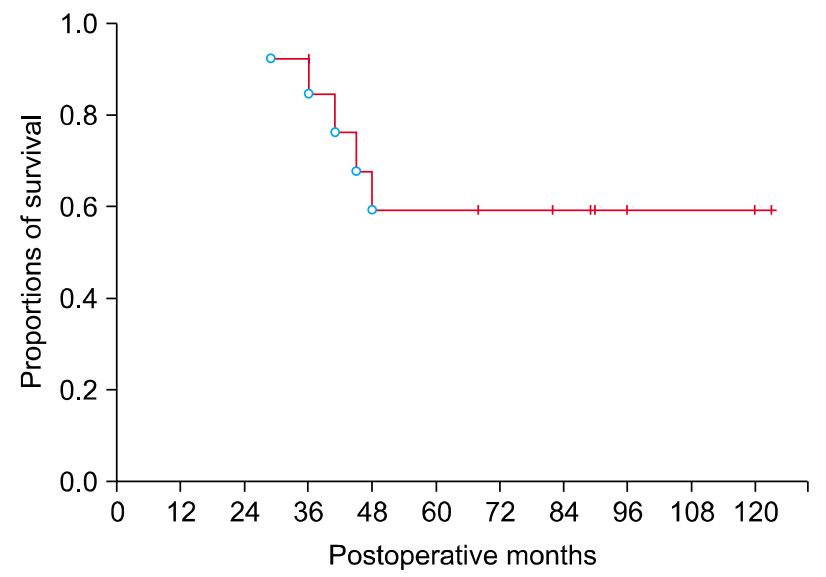

Fig. 2. Overall patient survival curves of 13 patients with malignant lesions. therapy were performed in 3. Their 1-year, 3-year, and 5 -year survival rates were $100 \%, 84.6 \%$, and $59.2 \%$, respectively (Fig. 2).

\section{DISCUSSION}

Intrahepatic IPNB is a rare condition, sporadically around the world. ${ }^{10-13}$ PNBs have been considered as a precursor lesion of cholangiocarcinoma, ${ }^{3,4}$ and its preoperative diagnosis is often difficult. The most common clinical manifestations of patients with IPNB were: recurrent abdominal pain, repeated episodes of acute cholangitis, and obstructive jaundice, as shown in this study.

The common abnormal finding of US and CT scans in intrahepatic IPNB patients was the intrahepatic duct 
dilatation. When the intraductal masses were not detected on US or CT scan, they were diagnosed as biliary stones, clonorchiasis, and benign biliary strictures. ERCP may be useful in making the diagnosis of IPNB. The characteristic ERCP findings of IPNB are multiple small filling defects and the serrated irregularity of the bile duct wall. On the cholangiography, diffuse bile duct dilatation and amorphous filling defects in the bile duct are characteristic. However, a large amount of mucin secretion and obstruction by the tumour prevent complete opacification of the entire biliary tract. As a result, the precise evaluation of the ductal extent by ERCP is often suboptimal. ${ }^{9,14}$

Cholangioscopic evaluation provided detailed information regarding the extent of disease, which enabled the appropriate surgical treatment to be provided to the patients. PTCS evaluation has some advantages over the conventional radiologic imaging. It may visualize the bile duct mucosa directly and detect small or subtle mucosal lesions that are not evident in direct cholangiogram. Because small papillary lesions may not be detected by conventional radiologic methods, these undetected lesions, usually remote from the main tumor, may be the foci of recurrence. We prefer PTCS to peroral cholangioscopy, because peroral cholangioscopic examination may be inferior to PTCS for complete evaluation of the intrahepatic duct, due to the difficulty in using the remote control of the babyscope. Therefore, PTCS examination is an indispensable preoperative procedure for determining the treatment modalities and the appropriate extent of resection in intrahepatic IPNB. It is also useful in patients with mucin-producing lesions, because mucin is observed as filling defects on direct cholangiography. ${ }^{9,14}$

Intrahepatic IPNB should not be regarded as a benign disease with low malignant potential, but rather a premalignant lesion with high malignant potential. IPNB with different malignant potentials can be finally diagnosed as adenomas, borderline tumour, non-invasive carcinoma, or invasive carcinoma in the literature, ${ }^{8}$ pointing to that the entire spectrum of IPNB represents a continuum of intraductal neoplastic progression. The pathogenesis of progression from benign to malignant disease may follow the adenomacarcinoma sequence. Although clinical presentations and gastroenteric metaplasia were somewhat different, the long-term survival rate was not found to be sig- nificantly different between the patients with NMBP and MBP. Because patients with intrahepatic IPNB have better prognosis than patients with usual cholangiocarcinoma, the surgical resection can be curative. Therefore, early and accurate diagnosis is important in this disease.

We concluded that intrahepatic IPNB is a rare type of biliary neoplasm which includes a histological spectrum ranging from benign disease to invasive malignancy. The long-term survival was anticipated after the complete curative resection. Therefore, early and accurate diagnosis is important for IPNB.

\section{REFERENCES}

1. Itatsu K, Zen Y, Ohira S, et al. Immunohistochemical analysis of the progression of flat and papillary preneoplastic lesions in intrahepatic cholangiocarcinogenesis in hepatolithiasis. Liver Int 2007;27:1174-1184.

2. Jang KT, Hong SM, Lee KT, et al. Intraductal papillary neoplasm of the bile duct associated with Clonorchis sinensis infection. Virchows Arch 2008;453:589-598.

3. Nakanuma Y, Sato Y, Harada K, et al. Pathological classification of intrahepatic cholangiocarcinoma based on a new concept. World J Hepatol 2010;2:419-427.

4. Zen Y, Fujii T, Itatsu K, et al. Biliary papillary tumors share pathological features with intraductal papillary mucinous neoplasm of the pancreas. Hepatology 2006;44:1333-1343.

5. Shimonishi T, Sasaki M, Nakanuma Y. Precancerous lesions of intrahepatic cholangiocarcinoma. J Hepatobiliary Pancreat Surg 2000;7:542-550.

6. Nakanuma Y. A novel approach to biliary tract pathology based on similarities to pancreatic counterparts: is the biliary tract an incomplete pancreas? Pathol Int 2010;60:419-429.

7. Kloek JJ, van der Gaag NA, Erdogan D, et al. A comparative study of intraductal papillary neoplasia of the biliary tract and pancreas. Hum Pathol 2011;42:824-832.

8. Naito $\mathrm{Y}$, Kusano $\mathrm{H}$, Nakashima $\mathrm{O}$, et al. Intraductal neoplasm of the intrahepatic bile duct: clinicopathological studyof 24 cases. World J Gastroenterol 2012;18:3673-3680.

9. Lee SS, Kim MH, Lee SK, et al. Clinicopathologic review of 58 patients with biliary papillomatosis. Cancer 2004;100:783-793.

10. Wu SD, Lu CD, Lu CJ, et al. Mucin-producing intrahepatic biliary papillomatosis. Surg Today 2010;40:845-850.

11. Nakanuma Y, Zen Y, Harada K, et al. Tumorigenesis and phenotypic characteristics of mucin-producing bile duct tumors: an immunohistochemical approach. J Hepatobiliary Pancreat Sci 2010; 17:211-222.

12. Nakanishi Y, Zen Y, Hirano S, et al. Intraductal oncocytic papillary neoplasm of the bile duct: the first case of peribiliary gland origin. J Hepatobiliary Pancreat Surg 2009;16:869-873.

13. Ohtsuka M, Kimura F, Shimizu H, et al. Similarities and differences between intraductal papillary tumors of the bile duct with and without macroscopically visible mucin secretion. Am J Surg Pathol 2011;35:512-521.

14. Yang J, Wang W, Yan L. The clinicopathological features of intraductal papillary neoplasms of the bile duct in a Chinese population. Dig Liver Dis 2012;44:251-256. 